Article

\title{
Closed Form Representations of Some Series in Darling's Model for Squeeze Film Damping with a Rectangular Plate
}

\author{
Martin Gugat \\ Department Mathematik, Friedrich-Alexander Universität Erlangen-Nürnberg, Cauerstr. 11, 91058 \\ Erlangen, Germany; E-Mail: gugat@am.uni-erlangen.de; Tel.: +49-9131-85-67130; \\ Fax: +49-9131-85-67134
}

Received: 25 April 2012; in revised form: 11 May 2012 / Accepted: 14 May 2012 /

Published: 21 May 2012

\begin{abstract}
Compressible squeeze film damping is a phenomenon of great importance for micromachines. For example, for the optimal design of an electrostatically actuated micro-cantilever mass sensor that operates in air, it is essential to have a model for the system behavior that can be evaluated efficiently. An analytical model that is based upon a solution of the linearized Reynolds equation has been given by R.B. Darling. In this paper we explain how some infinite sums that appear in Darling's model can be evaluated analytically. As an example of applications of these closed form representations, we compute an approximation for the critical frequency where the spring component of the reaction force on the microplate, due to the motion through the air, is equal to a certain given multiple of the damping component. We also show how some double series that appear in the model can be reduced to a single infinite series that can be approximated efficiently.
\end{abstract}

Keywords: squeeze film; optimal sensor design; resonant mass sensor; double series; efficient approximation of double series; evaluation of infinite series; Darling's model

\section{Introduction}

An analytical model for squeeze film damping is presented by Darling et al. in [2] for different venting conditions. Based upon the solution of the linearized Reynolds equations, series representations of the resulting reaction forces are presented for different air venting boundary 
conditions; in particular, for ideally vented rectangular vibrating plates. In [1], based upon the model of Darling et al. [2], an optimal sensor design problem is studied.

For optimal sensor design problems, the aim is to find system parameters for which a certain real valued function that measures the sensor's performance is maximized. In order to determine such parameters that solve the optimal design problems, it is useful to have a compact analytical model for the system behavior.

In [1], two kinds of boundary conditions appear for rectangular plates: Plates with two opposite edges closed and two opposite edges venting and plates with two adjacent edges venting and two adjacent edges closed. For the former, we give a closed form solution of the corresponding spring and damping constants that are given as infinite series in [1] and [2]. This model has also been used in [3] to model squeeze film effects. For the latter case, we present an approximation of the resulting reaction forces solution that can be evaluated efficiently. We also give a bound for the approximation error.

Let us start with a short presentation of the spring and damping constants for a rectangular plate with two opposite edges venting. Let $\mathrm{b}$ denote the plate dimension in the direction of venting and let $\mathrm{A}$ denote the plate area. $\mathrm{P}_{\mathrm{A}}$ is the ambient pressure and $\mathrm{g}_{0}$ is the nominal gap. Let $\mu$ denote the viscosity of the trapped gas. Define $\mathrm{k}_{\mathrm{n}}=\mathrm{n} \pi / \mathrm{b}$ and the constant

$$
\alpha^{2}=12 \mu /\left(g_{0}^{2} P_{a}\right)
$$

For the vibration frequency $\omega$, according to Darling's model we have the spring constant

$$
\begin{gathered}
\mathrm{k}=\left(8 \omega^{2} \mathrm{AP} \mathrm{A}_{\mathrm{A}} /\left(\boldsymbol{\pi}^{2} \mathrm{~g}_{0}\right)\right)\left[1 /\left[\omega^{2}+\left(\mathrm{k}_{1} / \alpha\right)^{4}\right]+1 /\left[3^{2}\left(\omega^{2}+\left(\mathrm{k}_{3} / \alpha\right)^{4}\right)\right]+1 /\left[5^{2}\left(\omega^{2}+\left(\mathrm{k}_{5} / \alpha\right)^{4}\right)\right]+\ldots\right] \\
=\left(8 \omega^{2} \mathrm{AP}_{\mathrm{A}} /\left(\boldsymbol{\pi}^{2} \mathrm{~g}_{0}\right)\right)\left(\alpha^{4} \mathrm{~b}^{4} / \boldsymbol{\pi}^{4}\right)\left[1 /\left[\alpha^{4} \mathrm{~b}^{4} \omega^{2} / \boldsymbol{\pi}^{4}+1\right]+1 /\left[3^{2}\left(\alpha^{4} \mathrm{~b}^{4} \omega^{2} / \boldsymbol{\pi}^{4}+3^{4}\right)\right]+1 /\left[5^{2}\left(\alpha^{4} \mathrm{~b}^{4} \omega^{2} / \boldsymbol{\pi}^{4}+5^{4}\right)\right]+\ldots\right]
\end{gathered}
$$

and the damping constant

$$
\begin{aligned}
\beta & =\left(8 \mathrm{AP}_{\mathrm{A}} /\left(\boldsymbol{\pi}^{2} \alpha^{2} \mathrm{~g}_{0}\right)\right)\left[\mathrm{k}_{1}^{2} /\left[\omega^{2}+\left(\mathrm{k}_{1} / \alpha\right)^{4}\right]+\mathrm{k}_{3}^{2} /\left[3^{2}\left(\omega^{2}+\left(\mathrm{k}_{3} / \alpha\right)^{4}\right)\right]+\mathrm{k}_{5}^{2} /\left[5^{2}\left(\omega^{2}+\left(\mathrm{k}_{5} / \alpha\right)^{4}\right)\right]+\ldots\right] \\
& =\left(8 \mathrm{AP}_{\mathrm{A}} /\left(\boldsymbol{\pi}^{2} \alpha^{2} \mathrm{~g}_{0}\right)\right)\left(\alpha^{4} \mathrm{~b}^{2} / \boldsymbol{\pi}^{2}\right)\left[1 /\left[\alpha^{4} \mathrm{~b}^{4} \omega^{2} / \boldsymbol{\pi}^{4}+1^{4}\right]+1 /\left[\left(\alpha^{4} \mathrm{~b}^{4} \omega^{2} / \boldsymbol{\pi}^{4}+3^{4}\right)\right]+1 /\left(\alpha^{4} \mathrm{~b}^{4} \omega^{2} / \boldsymbol{\pi}^{4}+5^{4}\right)+\ldots\right]
\end{aligned}
$$

In the sequel, we will give compact representations for $\mathrm{k}$ and $\beta$ that do not require the evaluation of infinite series. In order to do this, we introduce some auxiliary functions in the next section.

\section{Closed form Representations for Some Infinite Series}

In this section we define some auxiliary functions that appear in Darling's model as infinite series. In Section 4, we will show how stiffness and damping can be expressed using these series. For these series we present closed form representations that can be evaluated efficiently and thus are useful for the solution of the optimal design problem. For a real number $\mathrm{x}$ let

$$
\mathrm{F}(\mathrm{x})=1 /\left(1+\mathrm{x}^{4}\right)+1 /\left(3^{4}+\mathrm{x}^{4}\right)+1 /\left(5^{4}+\mathrm{x}^{4}\right)+1 /\left(7^{4}+\mathrm{x}^{4}\right)+\ldots
$$

then for all $\mathrm{x}$ unequal to zero we have the representation (with $\mathrm{w}(\mathrm{x})=\boldsymbol{\pi} \mathrm{x} / 2^{1 / 2}$ )

$$
\mathrm{F}(\mathrm{x})=2^{1 / 2} \pi[\sinh (\mathrm{w}(\mathrm{x}))-\sin (\mathrm{w}(\mathrm{x}))] /\left[8 \mathrm{x}^{3}[\cosh (\mathrm{w}(\mathrm{x}))+\cos (\mathrm{w}(\mathrm{x}))]\right]
$$

and $\mathrm{F}(0)=\boldsymbol{\pi}^{4} / 96$, which is also the maximal value attained by $F$. 
As a second real function, define

$$
\mathrm{B}(\mathrm{x})=1 /\left[1+\mathrm{x}^{4}\right]+1 /\left[3^{2}\left(3^{4}+\mathrm{x}^{4}\right)\right]+1 /\left[5^{2}\left(5^{4}+\mathrm{x}^{4}\right)\right]+1 /\left[7^{2}\left(7^{4}+\mathrm{x}^{4}\right)\right]+\ldots
$$

then we have for all $\mathrm{x}$ unequal to zero

$$
\mathrm{B}(\mathrm{x})=\boldsymbol{\pi}^{2} /\left(8 \mathrm{x}^{4}\right)-2^{1 / 2} \pi[\sinh (\mathrm{w}(\mathrm{x}))+\sin (\mathrm{w}(\mathrm{x}))] /\left[8 \mathrm{x}^{5}[\cosh (\mathrm{w}(\mathrm{x}))+\cos (\mathrm{w}(\mathrm{x}))]\right]
$$

and $\mathrm{B}(0)=\boldsymbol{\pi}^{4} / 960$, which is also the maximal value attained by $\mathrm{B}$. Note that Equation (4) implies that $\mathrm{x}^{4} \mathrm{~B}(\mathrm{x})$ converges to the limit $\boldsymbol{\pi}^{2} / 8$ as $\mathrm{x}$ tends to infinity.

For a complex number $\mathrm{z}$ with $-\mathrm{z}^{2}$ not equal to the square of any odd integer, let

$$
\mathrm{H}(\mathrm{z})=1 /\left[1+\mathrm{z}^{2}\right]+1 /\left[3^{2}\left(3^{2}+\mathrm{z}^{2}\right)\right]+1 /\left[5^{2}\left(5^{2}+\mathrm{z}^{2}\right)\right]+1 /\left[7^{2}\left(7^{2}+\mathrm{z}^{2}\right)\right]+\ldots
$$

then for all $\mathrm{z}$ unequal to zero we have the representation

$$
\mathrm{H}(\mathrm{z})=\boldsymbol{\pi}^{2} /\left(8 \mathrm{z}^{2}\right)-\boldsymbol{\pi} \tanh (\pi \mathrm{z} / 2) /\left(4 \mathrm{z}^{3}\right)
$$

and $\mathrm{H}(0)=\mathrm{F}(0)=\pi^{4} / 96$.

To prove Equation (6), the series Equation (5) can be interpreted as the Laplace-transform of the corresponding Fourier series. The sum of the Fourier series can be determined explicitly (see for example in reference [4], p. 900, For $0<x<\pi$ we have $\left.\sin x+\sin (3 x) / 3^{3}+\sin (5 x) / 5^{3}+\ldots=\pi x(\pi-x) / 8\right)$. Then $\mathrm{H}(\mathrm{z})$ can be obtained as the Laplace transform of the $2 \pi$-periodic function given by the sum of the Fourier series. Note that if $z^{2}=j \omega$ is purely imaginary, we have

$$
\mathrm{H}(\mathrm{z})=\mathrm{F}\left(|\omega|^{1 / 2}\right)-\mathrm{j} \omega \mathrm{B}\left(|\omega|^{1 / 2}\right)
$$

that is $F\left(|\omega|^{1 / 2}\right)$ is the real part of $H(z)$ and the imaginary part of $H(z)$ is given by $-\omega B\left(|\omega|^{1 / 2}\right)$. This allows deriving Equations (4) and (2) from (6).

\section{Efficient Approximation of a Double Series}

For certain boundary conditions, in Darling's model infinite double series appear. We give approximations for these double series that can be evaluated efficiently. We also provide bounds for the approximation error. Let the parameter $\lambda$ greater than or equal to 1 be given.

For a complex number $\mathrm{z}$, let $\mathrm{D}$ denote the double series

$$
\mathrm{D}(\mathrm{z})=\Sigma_{\mathrm{nm}} \mathrm{d}(\mathrm{n}, \mathrm{m})=\mathrm{d}(1,1)+\mathrm{d}(1,2)+\mathrm{d}(2,1)+\mathrm{d}(3,1)+\mathrm{d}(2,2)+\mathrm{d}(1,3)+\ldots
$$

with

$$
\mathrm{d}(\mathrm{n}, \mathrm{m})=1 /\left[(2 \mathrm{n}-1)^{2}(2 \mathrm{~m}-1)^{2}\left(\lambda^{2}(2 \mathrm{n}-1)^{2}+(2 \mathrm{~m}-1)^{2}+\mathrm{z}^{2}\right)\right]
$$

Using the definition of $\mathrm{H}$, we obtain the equation

$$
\mathrm{D}(\mathrm{z})=\mathrm{H}\left(\left(\lambda^{2}+\mathrm{z}^{2}\right)^{1 / 2}\right)+1 / 3^{2} \mathrm{H}\left(\left(\lambda^{2} 3^{2}+\mathrm{z}^{2}\right)^{1 / 2}\right)+1 / 5^{2} \mathrm{H}\left(\left(\lambda^{2} 5^{2}+\mathrm{z}^{2}\right)^{1 / 2}\right)+\ldots
$$

Define the auxiliary function $G(z)=\tanh (0.5 \pi z) / z$. Then $G(-z)=G(z)$, that is, $G$ is even. Using the representation (6) for $\mathrm{H}$, we obtain

$$
\begin{gathered}
\mathrm{D}(\mathrm{z})=\boldsymbol{\pi}^{2} /\left(8 \lambda^{2}\right) \mathrm{H}(\mathrm{z} / \lambda)- \\
-\pi / 4\left[\mathrm{G}\left(\left(\lambda^{2}+\mathrm{z}^{2}\right)^{1 / 2}\right) /\left(\lambda^{2}+\mathrm{z}^{2}\right)+\mathrm{G}\left(\left(\lambda^{2} 3^{2}+\mathrm{z}^{2}\right)^{1 / 2}\right) /\left(3^{2}\left(\lambda^{2} 3^{2}+\mathrm{z}^{2}\right)\right)\right. \\
\left.+\mathrm{G}\left(\left(\lambda^{2} 5^{2}+\mathrm{z}^{2}\right)^{1 / 2}\right) /\left(5^{2}\left(\lambda^{2} 5^{2}+\mathrm{z}^{2}\right)\right)+\ldots\right]
\end{gathered}
$$


Thus we have reduced the double series to a standard infinite series. As an approximation for D, we propose to use the function A defined as

$$
\begin{gathered}
\mathrm{A}(\mathrm{z})=\boldsymbol{\pi}^{2} /\left(8 \lambda^{2}\right) \mathrm{H}(\mathrm{z} / \lambda)-\pi / 4\left[\mathrm{G}\left(\left(\lambda^{2}+\mathrm{z}^{2}\right)^{1 / 2}\right) /\left(\lambda^{2}+\mathrm{z}^{2}\right)+\mathrm{G}\left(\left(\lambda^{2} 3^{2}+\mathrm{z}^{2}\right)^{1 / 2}\right) /\left(3^{2}\left(\lambda^{2} 3^{2}+\mathrm{z}^{2}\right)\right)\right. \\
\left.+\mathrm{G}\left(\left(\lambda^{2} 5^{2}+\mathrm{z}^{2}\right)^{1 / 2}\right) /\left(5^{2}\left(\lambda^{2} 5^{2}+\mathrm{z}^{2}\right)\right)+\ldots+\mathrm{G}\left(\left(\lambda^{2}(2 \mathrm{k}-1)^{2}+\mathrm{z}^{2}\right)^{1 / 2}\right) /\left((2 \mathrm{k}-1)^{2}\left(\lambda^{2}(2 \mathrm{k}-1)^{2}+\mathrm{z}^{2}\right)\right)\right]
\end{gathered}
$$

with a natural number $\mathrm{k}$. Then we have $\mathrm{D}(\mathrm{z})=\mathrm{A}(\mathrm{z})-\mathrm{R}(\mathrm{z}, \mathrm{k})$ with the remainder term $\mathrm{R}(\mathrm{z}, \mathrm{k})$.

If $z^{2}$ is purely imaginary, we have $\left|\lambda^{2}(2 n+1)^{2}+z^{2}\right| \geq \lambda^{2}(2 n+1)^{2}>1$.

Moreover, $\mid 1+\exp \left(-\pi\left(\lambda^{2}(2 \mathrm{n}+1)^{2}+\mathrm{z}^{2}\right)^{1 / 2} \mid>0.5\right.$. Since $\tanh (0.5 \pi \mathrm{z})=-1+2 /(1+\exp (-\pi \mathrm{z}))$ this implies

$$
\left|\mathrm{G}\left(\left(\lambda^{2}(2 \mathrm{n}+1)^{2}+\mathrm{z}^{2}\right)^{1 / 2}\right)\right|=\left|\left[-1+2 /\left(1+\exp \left(-\pi\left(\lambda^{2}(2 \mathrm{n}+1)^{2}+\mathrm{z}^{2}\right)^{1 / 2}\right)\right)\right] /\left(\lambda^{2}(2 \mathrm{n}+1)^{2}+\mathrm{z}^{2}\right)^{1 / 2}\right|<5 /(\lambda(2 \mathrm{n}+1))
$$

and hence

$$
|\mathrm{R}(\mathrm{z}, \mathrm{k})|<5 \pi /\left(4 \lambda^{3}\right)\left[1 /(2 \mathrm{k}+1)^{5}+1 /(2 \mathrm{k}+3)^{5}+1 /(2 \mathrm{k}+5)^{5}+1 /(2 \mathrm{k}+7)^{5}+\ldots\right]
$$

For $\mathrm{k}=7$, we obtain $|\mathrm{R}(\mathrm{z}, 7)|<2 \times 10^{-5} / \lambda^{3}$. Thus for $\mathrm{k}=7$ and purely imaginary $\mathrm{z}^{2}, \mathrm{~A}$ approximates $\mathrm{D}$ with a uniform bound that is less than $2 \times 10^{-5}$.

\section{Computation of the Spring and Damping Constants: Two Opposite Edges Venting}

The spring and damping constants for a plate with two opposite edges venting can be expressed in terms of the functions F and B that have been introduced in Section 2 in Equations (2) and (4). According to Darling's model, we have (see Li and Miller [1]) the spring constant

$$
\mathrm{k}=\left(8 \omega^{2} \mathrm{AP} \mathrm{P}_{\mathrm{A}} /\left(\boldsymbol{\pi}^{2} \mathrm{~g}_{0}\right)\right)\left(\alpha^{4} \mathrm{~b}^{4} / \boldsymbol{\pi}^{4}\right) \mathrm{B}\left(\alpha \mathrm{b} \omega^{1 / 2} / \boldsymbol{\pi}\right)
$$

with the notation introduced in Section 1. The dimension in Equation (12) is $\mathrm{N} / \mathrm{m}$. The damping constant $\beta$ is given by

$$
\beta=\left(8 \mathrm{AP} A /\left(\pi^{2} \alpha^{2} \mathrm{~g}_{0}\right)\right)\left(\alpha^{4} \mathrm{~b}^{2} / \pi^{2}\right) \mathrm{F}\left(\alpha \mathrm{b} \omega^{1 / 2} / \boldsymbol{\pi}\right)
$$

The dimension in Equation (13) is $\mathrm{N} \mathrm{s} / \mathrm{m}$. Using Equations (4) and (2), these functions can easily be implemented for numerical evaluation.

For a sensor design, Li and Miller [1] consider a square plate of side length $\mathrm{p}$ with a square hole of side length $\mathrm{s}$ in the middle. This plate is divided into eight elements. Four plates with opposite edges venting appear as side elements. The other four elements are square corner plates with adjacent edges venting. The elements with opposite venting are described by Equations (12) and (13), where the value of $b$ is given by $(p-s) / 2$. The optimal design problem consists in determining an optimal value for $s$. For the corner elements with adjacent edges venting the side length is $(p-s) / 2$. The corresponding spring and damping constants are given by double series that are more difficult to evaluate analytically.

We will give a suitable approximation in Section 5. For the elements with opposite venting described by Equations (12) and (13) according to the Equation (46) in reference [3], the reaction force on the plate due to motion through the air is

$$
\text { Force }=C_{0} j \omega H\left(\left(\alpha b \omega^{1 / 2} / \boldsymbol{\pi}\right)(1+j) / 2^{1 / 2}\right)
$$

where $\mathrm{C}_{0}$ is a suitably chosen real constant, $\mathrm{j}^{2}=-1$ and $\mathrm{H}$ can be computed using Equation (6). 


\subsection{An Application: Approximation of the Critical Frequency where $k=\gamma \beta$}

Our representations allow the approximate computation of the frequency for which $\mathrm{k}=\gamma \beta$, where $\gamma$ is a given constant with the appropriate dimensions. At this frequency, the ratio $\mathrm{k} / \beta$ of viscous force and spring force is equal to the given value $\gamma$. According to Equations (12) and (13), this problem is equivalent to the solution of the equation

$$
\mathrm{x}^{4} \mathrm{~B}(\mathrm{x})=\mathrm{CF}(\mathrm{x})
$$

with $\mathrm{x}=\alpha \mathrm{b} \omega^{1 / 2} / \boldsymbol{\pi}$ and the dimensionless constant $\mathrm{C}=\gamma \alpha^{2} \mathrm{~b}^{2} / \boldsymbol{\pi}^{2}$. Equation (15) is equivalent to

$$
\cosh (\mathrm{w}(\mathrm{x}))+\cos (\mathrm{w}(\mathrm{x}))=\left(2^{1 / 2} / \pi\right)\left[\left(1 / \mathrm{x}+\mathrm{C} / \mathrm{x}^{3}\right) \sinh (\mathrm{w}(\mathrm{x}))+\left(1 / \mathrm{x}-\mathrm{C} / \mathrm{x}^{3}\right) \sin (\mathrm{w}(\mathrm{x}))\right]
$$

If we let $\mathrm{w}=\mathrm{w}(\mathrm{x})$ and $\mathrm{x}=\left(2^{1 / 2} / \pi\right) \mathrm{w}$, we obtain the equation

$$
\cosh (\mathrm{w})+\cos (\mathrm{w})-\sinh (\mathrm{w}) / \mathrm{w}-\sin (\mathrm{w}) / \mathrm{w}=\left(\pi^{2} \mathrm{C} / 2\right)[\sinh (\mathrm{w})-\sin (\mathrm{w})] / \mathrm{w}^{3}
$$

If we replace the functions by the corresponding power series, this yields the equation

$$
\mathrm{w}^{4} / 15+\mathrm{w}^{8} / 22680+\ldots . .=\left(\boldsymbol{\pi}^{2} \mathrm{C} / 2\right)\left[1 / 3+\mathrm{w}^{4} / 2520+\mathrm{w}^{8} / 19958400+\ldots\right]
$$

By considering only the constant term and the terms with $\mathrm{w}^{4}$ we obtain the approximation

$$
\mathrm{W}=\left[\boldsymbol{\pi}^{2} \mathrm{C} /\left(0.4-\boldsymbol{\pi}^{2} \mathrm{C} / 840\right)\right]^{1 / 4}
$$

For Equation (15) this yields the approximate solution

$$
\mathrm{x}=\left(2^{1 / 2} / \boldsymbol{\pi}\right)\left[\boldsymbol{\pi}^{2} \mathrm{C} /\left(0.4-\boldsymbol{\pi}^{2} \mathrm{C} / 840\right)\right]^{1 / 4}
$$

If we replace the denominator $\left(0.4-\pi^{2} \mathrm{C} / 840\right)$ by 0.4 , this yields the critical frequency

$$
\omega=\left[2 \pi /\left(\alpha^{2} b^{2}\right)\right][C / 0.4]^{1 / 2}
$$

Example With $\mathrm{C}=2 \times 10^{-7}$, Equation (17) has the solution $\mathrm{w}=0.0471321702447414 \ldots$ The approximation $\mathrm{w}_{0}=\left[\boldsymbol{\pi}^{2} \mathrm{C} / 0.4\right]^{1 / 4}$ yields the value $\mathrm{w}_{0}=\boldsymbol{\pi}^{1 / 2} /\left(2^{1 / 4} 10^{1 / 2}\right)=0.0471321702139757 \ldots$ Moreover, we have $\mathrm{w}_{1}=\left[\boldsymbol{\pi}^{2} \mathrm{C} /\left(0.4-\boldsymbol{\pi}^{2} \mathrm{C} / 840\right)\right]^{1 / 4}=0.04713217028319835 \ldots$.

\section{Computation of the Reaction Force: Adjacent Edges Venting}

According to Darling's model, the normalized reaction force on a rectangular plate with two adjacent edges venting can be expressed as a double series. Define

$$
\mathrm{k}_{\mathrm{mn}}=\left((2 \mathrm{~m}-1)^{2} \pi^{2} /\left(4 \mathrm{a}^{2}\right)+(2 \mathrm{n}-1)^{2} \pi^{2} /\left(4 \mathrm{~b}^{2}\right)\right)^{1 / 2}
$$

then from Equation (19) in [2] we obtain

$$
\begin{gathered}
F(t) /\left(a b P_{a}\right)=-64 j \omega \exp (j \omega t)\left(H^{\prime} \eta / \pi^{4}\right)[u(1,1)+u(1,2)+u(2,1)+u(3,1)+u(2,2)+u(1,3)+u(1,4)+ \\
u(2,3)+u(3,2)+u(4,1)+\ldots]
\end{gathered}
$$

where $\mathrm{u}(\mathrm{m}, \mathrm{n})=\left\{1 /\left[(2 \mathrm{~m}-1)^{2}(2 \mathrm{n}-1)^{2}\right]\right\}\left\{1 /\left[\mathrm{j} \omega+\left(\mathrm{k}_{\mathrm{mn}} / \alpha\right)^{2}\right]\right\}$. Here $\mathrm{H}^{\prime}$ is a constant giving the normalized amplitude of the plate vibration. For an isothermal process $\eta=1$, while for an adiabatic process $\eta$ is the quotient of the specific heats. From Equation (7) we get

$$
\mathrm{F}(\mathrm{t}) /\left(\mathrm{abP} \mathrm{P}_{\mathrm{a}}\right)=-64 \mathrm{j \omega} \exp (\mathrm{j} \mathrm{t})\left(\mathrm{H}^{\prime} \eta / \pi^{4}\right)\left(4 \mathrm{a}^{2} \alpha^{2} / \boldsymbol{\pi}^{2}\right) \mathrm{D}\left(2 \mathrm{a} \alpha(j \omega)^{1 / 2} / \pi\right) \text { with } \lambda=\mathrm{a} / \mathrm{b}
$$


for the argument $\mathrm{z}=2 \mathrm{a} \alpha(\mathrm{j} \omega)^{1 / 2} / \pi$ of $\mathrm{D}$ the number $\mathrm{z}^{2}$ is purely imaginary. Therefore for a numerical approximation of $\mathrm{F}(\mathrm{t}) /\left(\mathrm{abP}_{\mathrm{a}}\right)$, the function

$$
\text { Force }_{\text {approx }}(\mathrm{t})=-64 j \omega \exp (j \omega \mathrm{t})\left(\mathrm{H}^{\prime} \eta / \pi^{4}\right)\left(4 \mathrm{a}^{2} \alpha^{2} / \pi^{2}\right) \mathrm{A}\left(2 \mathrm{a} \alpha(j \omega)^{1 / 2} / \pi\right)
$$

(with $\lambda=\mathrm{a} / \mathrm{b}$ assuming that $\lambda \geq 1$ ) can be used. For $\mathrm{k}=7$, the approximation error is uniformly bounded by $2 \times 10^{-5}$. Since for the evaluation of $H$ that appears in the definition of $A$, the representation given in Equation (6) can be used, a direct numerical evaluation of the function Force $_{\text {approx }}(t)$ is possible.

\section{Conclusions}

The air between two parallel microplates can compress to store energy or vent to dampen energy. This compressible squeeze film damping is a phenomenon of great importance for micromachines. In reference [2], Darling et al have presented an analytical model for this phenomenon that is based upon expansions in series of eigenfunctions. To apply this model for optimization purposes, it is useful to have closed form representations of the corresponding functions. In this paper we give such a representation for the case that two opposite edges of the plate are closed. The case where two adjacent edges of the plate are closed, leads to functions given by double series. For this double series we provide an approximation that can be computed easily. Moreover, we give a bound for the corresponding approximation error.

\section{References}

1. Li, C.; Miller, M.H. Optimization Strategy for Resonant Mass Sensor Design in the Presence of Squeeze Film Damping. Micromachines 2010, 1, 112-128.

2. Darling, R.B.; Hivick, C.; Xu, J. Compact Analytical Modeling of Squeeze Film Damping with Arbitrary Venting Conditions Using a Green's Function Approach. Sens. Actuat. A 1998, 70, 32-41.

3. Miller, M.H.; Perrault, J.A.; Parker, G.G.; Bettig, B.P.; Bifano, T.G. Simple Models for Piston-Type Micromirror Behavior. J. Micromech. Microeng. 2006, 16, 303-313.

4. Bronstein, I.N.; Semendjajew, K.A.; Musiol, G.; Mühlig, H. Taschenbuch der Mathematik, Verlag Harri Deutsch, Frankfurt am Main, Germany, 1995.

(C) 2012 by the authors; licensee MDPI, Basel, Switzerland. This article is an open access article distributed under the terms and conditions of the Creative Commons Attribution license (http://creativecommons.org/licenses/by/3.0/). 